\title{
A novel acemannan-chitosan modified lipid nanoparticles as intracellular delivery vehicles of antibiotic
}

\author{
Tri Suciati ${ }^{1 *}$, Putriana Rachmawati ${ }^{1}$, Eldi Soraya ${ }^{1}$, Andhika B. Mahardhika ${ }^{2}$, Satrialdi ${ }^{1}$, Rika Hartarti ${ }^{3}$, Kusnandar Anggadiredja $^{4}$ \\ ${ }^{1}$ Department of Pharmaceutics, School of Pharmacy, Bandung Institute of Technology, Bandung, Indonesia. \\ ${ }^{2}$ Department of Pharmacochemistry, School of Pharmacy, Bandung Institute of Technology, Bandung, Indonesia. \\ ${ }^{3}$ Department of Pharmaceutical Biology, School of Pharmacy, Bandung Institute of Technology, Bandung, Indonesia. \\ ${ }^{4}$ Department of Pharmacology and Clinical Pharmacy, School of Pharmacy, Bandung Institute of Technology, Bandung, Indonesia.
}

\section{ARTICLE INFO \\ Received on: 01/09/2018 \\ Accepted on: 17/11/2018 \\ Available online: 30/12/2018}

Key words:

Intracellular infection, lipid

nanoparticles, acemannan-

chitosan conjugate,

rifampicin.

\begin{abstract}
Killing intracellular pathogens is often hampered by poor antibiotic penetration. A nanoparticle formulation with surface properties similar to microbial particles can be advantageous as a carrier for intracellular delivery of antimicrobial agents. Surface-modified lipid nanoparticles with chitosan-conjugated acemannan - an acetylated polymannose of aloe gel isolate, have been proposed to deliver rifampicin intracellularly. Chitosan-acemannan (COS$\mathrm{ACE}$ ) conjugate was bound electrostatically to rifampicin-loaded nanostructured lipid carrier (NLC) to form COSACE lipid nanoparticles. The nanoparticles showed a diameter size of around $300 \mathrm{~nm}$ at a low polydispersity index, and positive zeta potential of $1.66 \pm 1.39 \mathrm{mV}$. Transmission electron microscope and confocal images showed that the COS-ACE lipid nanoparticles were spherical in shape with NLC in the center surrounded by COS-ACE conjugate. Rifampicin was highly entrapped within lipid nanoparticles and released from the particles at $\mathrm{pH} 5$ at higher rate compared with $\mathrm{pH}$ 7.4. The lyophilized nanoparticles were stable at the storage temperature of $4{ }^{\circ} \mathrm{C}$ and $25^{\circ} \mathrm{C}$, in terms of the content and particle size. Furthermore, the COS-ACE lipid nanoparticles was non-toxic against Vero and $\mathrm{BALB} / \mathrm{c} 3 \mathrm{~T} 3$ cells and increased intracellular accumulation of lipid soluble antibiotic, suggesting its functionality in intracellular targeting.
\end{abstract}

\section{INTRODUCTION}

Infectious diseases caused by intracellular pathogens, such as endocarditis, tuberculosis, and meningitis, have been a global problem causing millions of deaths every year (Armstead and $\mathrm{Li}, 2011$ ). It becomes a challenge in antimicrobial therapy due to poor capability of antimicrobials to penetrate inside the target cells and the pathogen's ability to develop resistance against antimicrobials (Armstead and Li, 2011; Bhise et al., 2018; Xie et al., 2014). Furthermore, cases of multi-drug resistance (MDR) are now emerging and making intracellular infection treatments become more challenging.

"Corresponding Author

Tri Suciati, Department of Pharmaceutics, School of Pharmacy, Bandung Institute of Technology, Bandung, Indonesia.

E-mail:tri.suciati@fa.itb.ac.id
Rifampicin and its derivative are primarily an antitubercular agent, but it has also been reported to have activity against non-tuberculous mycobacteria, Staphylococcus aureus and fungal organisms (Chaubey and Mishra, 2014; Forrest and Tamura, 2010; Perlroth et al., 2008). With the current emergence of MDR bacteria, rifampicin combination therapies could possibly be used as an alternative, which may be beneficial for the treatment of meningitis, prosthetic joint infections, osteomyelitis, and endocarditis (Ellington et al., 2006; Faville et al., 1978; Forrest and Tamura, 2010). As shown by a study conducted by Forrest and Tamura, rifampin combination therapy may have a potential clinical benefit for patients with penicillin-resistant Pneumococcal meningitis. This is due to the ability of rifampin to reduce biofilm formation of $S$. aureus. The potential efficacy of rifampicin for the treatment of staphylococcal osteomyelitis was also reported in another study (Ellington et al., 2006; Jørgensen et al., 2017). When rifampicin was introduced to human osteoblasts, it 
significantly decreased the number of $S$. aureus within osteoblasts when compared to control. However, even though rifampicin combination therapy was effective against staphylococcal infections, resistance toward rifampicin still emerged (Forrest and Tamura, 2010). One of the most prominent approaches proposed to overcome this challenge is by encapsulating rifampicin in nanocarriers for intracellular-targeted delivery.

Over the decades, nanoparticles including polymeric nanoparticles, liposomes, dendrimers, nanostructured lipid carrier (NLC), and polymeric micelles have been explored for intracellular delivery of antimicrobial drugs that demonstrate a high bactericidal activity in vitro. Nanoparticles have been reported to improve cellular penetration, intracellular retention, and even avoid intracellular inactivation of antimicrobial drugs (Xie et al., 2014). Also, surface modification of nanocarriers with chitosan-acemannan conjugate has emerged as a strategy to enhance the cytoplasmic solubility of hydrophobic drugs, avoid off-target toxicity, minimize non-specific uptake, prolong circulation time, and allow for intracellular targeting (van Vlerken et al., 2007). Therefore, in this study, a novel acemanan-chitosan surface modified of NLC (COS-ACE lipid nanoparticles) loaded with rifampicin was developed for selective drug delivery to intracellular cells. By mimicking the invasion route of pathogens, acemannan and chitosan were used as the targeting agents to C-type lectin receptors (CLRs), which have been demonstrated as a potential entry receptor for various pathogens (Kerrigan and Brown, 2008; Neyrolles and Guilhot, 2011).

Mycobacteria can enter the host cell via various surface receptors including scavenger receptor, complement receptors, and C-type lectins, such as dendritic cell-specific intercellular adhesion molecule-3 grabbing non integrin (DC-SIGN) and the mannose receptor (MR) (Neyrolles and Guilhot, 2011; Schlesinger et al., 2008). Different glycolipids within the mycobacterial cell envelope function as key ligands for these receptors, allowing molecular docking between the pathogen and the cell that eventually leads to microbial invasion. Based on a study by Naglik and Moyes (2011), mannan molecules in fungal organisms, such as Candida albicans, can also be recognized by CLRs.

Chitosan and acemannan within the nanoparticles serve as glycolipids to target the CLR. Acemannan was reported to be able to recognize MR and DC-SIGN (Lee et al., 2001). Chitosan — which also has a broad antimicrobial spectrum - was conjugated with acemannan by using amine reducing agents (Goy et al., 2009). The COS-ACE conjugate was further bound to NLC through electrostatic interaction with the lipid and oil component to form self-assembled COS-ACE lipid nanoparticles. Rifampicin was selected as an antibiotic model based on its antimicrobial activity, hydrophobic characteristic, and potentially applied in various intracellular infections. Improving cell specificity and enhancing cellular uptake are the foremost objectives of the proposed drug delivery system.

\section{MATERIALS AND METHODS}

\section{Materials}

Rifampicin was donated by PT. Meprofarm (Indonesia), acemannan (BiAloe ${ }^{\circledR}$, LorAnd Laboratories, Houston, TX), low molecular weight chitosan (chitoolygosaccharide, COS ) was acid hydrolyzed from chitosan (Biotech Surindo, Indonesia), oleic acid, cetyl alcohol, and stearyl alcohol (PT. Ecogreen oleochemical, Indonesia), Tween ${ }^{\circledR} 80$ (Bratachem, Indonesia), Nile red, and sodium triacetoxyborohydride $\left[\mathrm{NaBH}(\mathrm{OAc})_{3}\right]$ were purchased from Sigma Aldrich (Singapore), acetic acid, tetrahydrofuran, Dulbecco's Modified Eagle Medium (DMEM), Heat inactivated foetal bovine serum (FBS), Alamar Blue ${ }^{\circledR}$ and toluidin blue were purchased from Gibco ${ }^{\mathrm{TM}}$ (Invitrogen, Indonesia SP), Vero cells and Balb/c 3T3 cells were obtained from American Type Culture Collection. All other chemicals were of reagent grade.

\section{Synthesis of chitosan-acemannan (COS-ACE) conjugate}

COS-ACE conjugate was prepared using the procedure modified from Abdel-Magid et al. (1996). Chitosan and sodium triacetoxyborohydride at the ratio of $3: 4$ were dissolved in tetrahydrofuran and stirred for 12 hours. The mixture was left in the fume hood at room temperature for another 12 hours to evaporate the solvent and to maximize the reduction of primary and secondary amine of chitosan. Acemannan solution in $1 \%$ acetic acid at $\mathrm{pH} 4$ was added to the reduced chitosan and stirred for 24 hours to conduct the reaction. The conjugate was provided by rotary evaporation at $60^{\circ} \mathrm{C}$ and $82 \mathrm{mBar}$ and kept in a vacuum dessicator until used. The COS-ACE conjugate was analyzed by infrared spectrophotometry (Shimadzu IR Prestige 21 ) to distinguish the difference peaks between chitosan and the conjugate. The molecular weight of the conjugate was determined using Ubbelohde-type capillary viscometer and calculated using Mark-Houwink-Sakurada equation (Kasaai et al., 2000).

$$
[\eta]=\mathrm{K} \cdot \mathrm{Mw} \cdot \alpha
$$

where $\eta$ is intrinsic viscosity (cps), Mw is Molecular weight $(\mathrm{kDa}), \mathrm{K}$ and $\alpha$ - the constants for solvent system used( 0.2 $\mathrm{M} \mathrm{NaCl}$ and $0.1 \mathrm{M}$ acetic acid $=1: 1)-$ are $1.8 \times 10^{-5}$ and 0.93 , respectively.

\section{Formulation of rifampicin loaded COS-ACE lipid nanopar- ticles}

Preparation of rifampicin-loaded nanostructured lipid carriers (NLC)

Rifampicin-loaded NLC was prepared by an emulsification-solvent evaporation method. Various variables were studied to produce particles with a size ranging from 200 to $500 \mathrm{~nm}$. The variables included lipid type, total lipid, surfactant and rifampicin concentrations, the speed and duration of homogenization, and also sonicator type. The oil phase was optimized consisting of ethyl acetate solution of rifampicin and an oleic acid blend with stearyl alcohol or cetyl alcohol at the ratio of $1: 1$ or $1: 2$, respectively. After the oil phase was dissolved completely in ethyl acetate, it was added dropwise to the aqueous phase containing Tween ${ }^{\circledR} 80$ as the surfactant. Both phases were emulsified by homogenization (Ultra Turrax IKA ${ }^{\circledR}$ T-25) operated at 8,000-10,000 rpm for 3, 5, and 10 minutes. Further, the emulsion was sonicated for 3 and 5 minutes using probe or water bath sonicators. Finally, the NLC was solidified by magnetic stirring for an hour using to ensure a complete evaporation of ethyl acetate. The obtained NLC was isolated by centrifugation at $10,000 \mathrm{rpm}$ for 30 minutes and further lyophilized. 
Optimization of COS-ACE lipid nanoparticles formation

A one-step of COS-ACE lipid nanoparticles production was employed by adding COS-ACE conjugate in the water phase during emulsification process of NLC preparation. In brief, COSACE conjugate and Tween ${ }^{\circledR} 80$ were dissolved in deionized water and emulsified with an oil phase comprising of ethyl acetate solution of rifampicin and lipid blend. Following emulsification process using homogenization and sonication, sodium tripolyphosphate was added dropwise up to $0.12 \% \mathrm{w} / \mathrm{v}$ of the total formulation. Finally, the solvent was evaporated and particle solidified by stirring for 1 hour. COS-ACE lipid nanoparticles was collected by centrifugation and lyophilization.

The selected lipid here was oleic acid-stearyl alcohol blend at 1:1 ratio based on the previous NLC optimization that could produce the smallest NLC particles. The amount of COSACE conjugate added in lipid nanoparticles formulation was varied according to the amount of total lipid used at the ratio of $1: 1$. Various parameters were optimized during COS-ACE lipid nanoparticles preparation, i.e the amount of total lipid and rifampicin, and also the duration and speed of homogenization and sonication for emulsification.

\section{Optimization of lyophilization process}

The optimum formulation of COS-ACE lipid nanoparticles was lyophilized overnight. The freezing step prior to lyophilization process was optimized to obtain the target size of particles, namely: freezing in the freezer $\left(-20^{\circ} \mathrm{C}\right)$, cold ethanol $\left(-40^{\circ} \mathrm{C}\right)$, and liquid nitrogen $\left(-196^{\circ} \mathrm{C}\right)$ (Chung et al., 2012). The lyophilized COS-ACE lipid nanoparticles was kept in a tight amber vial and stored in refrigerator until used.

\section{Physical characterization of COS-ACE lipid nanoparticles}

Particle size and polydispersity index of the lyophilized lipid nanoparticles were measured by photon correlation spectroscopy (DelsaTM C Nano Particle Analyzer, Coulter Beckman) after appropriate dilution with double distilled water. The surface charge of the particles was measured by Electrophoretic Light Scattering (Delsa ${ }^{\mathrm{TM}}$ Nano C particle Analyzer, Coulter Beckman). The morphology of COS-ACE lipid nanoparticles was observed on a transmission electron microscope (TEM Hitachi HT7700). The sample was homogenized in double distilled water $(0.02 \% \mathrm{w} / \mathrm{v})$ and $10 \mu \mathrm{l}$ of the sample was dropped onto the specimen film that had been attached to a carbon coated copper grid. The specimen was allowed to stand for 1 minute and the excess of the fluid on the grid was removed using a filter paper. The specimen was then air-dried for 30 minutes and the film was then scanned under a focused beam microscopy with accelerating voltage of $100 \mathrm{kV}$. Furthermore, lipid nanoparticles morphology was revealed using confocal microscope (CLSM Olympus FV1200 and Fluoview FV10i) by adding lipid soluble nile red fluorochrome.

\section{Evaluation of encapsulation efficiency and loading capacity of COS-ACE lipid nanoparticles}

The COS-ACE lipid nanoparticles were centrifuged at the speed of $13,000 \mathrm{rpm}$ for 45 minutes. The entrapment efficiency $(\% \mathrm{EE})$ and loading capacity $(\% \mathrm{LC})$ of the formulation were analyzed by indirect method and calculated by the following equations:

$$
\begin{aligned}
& \mathrm{EE}(\%)=\frac{\text { rifampicin added }- \text { free rifampicin }}{\text { rifampicin added }} \times 100 \\
& \mathrm{LC}(\%)=\frac{\text { weight of rifampicin }}{\text { weight of COS }- \text { ACE nanolipid }} \times 100
\end{aligned}
$$

\section{In-vitro rifampicin release}

The amount of rifampicin released from COS-ACE lipid nanoparticles particles were studied using a dialysis bag diffusion technique. A known amount of rifampicin-encapsulated COS-ACE lipid nanoparticles were loaded in dialysis bags (MWCO 1,000 $\mathrm{Da}$, Sigma, Singapore) and immersed in a receptor compartment containing $100 \mathrm{ml}$ of $\mathrm{PBS}$ at $\mathrm{pH} 5$ and 7.4 , at $37^{\circ} \mathrm{C}$ in the presence of ascorbic acid. Ascorbic acid was used to prevent degradation rifampicin in the dissolution medium due to atmospheric oxygen (Patel et al., 2013). At predetermined time intervals, $1 \mathrm{~mL}$ of samples was withdrawn and replaced with the same volume of fresh PBS. The samples were diluted with a water-methanol admixture and rifampicin concentration was measured by visible spectrophotometer at $475 \mathrm{~nm}$ (Kumar et al., 2008). The released drug (RC) was calculated using Equation (4).

$$
\mathrm{RC}(\%)=\frac{\text { weight of released rifampicin }}{\text { weight of encapsulated rifampicin }} \times 100 \%
$$

\section{Drug stability study}

Physical stability COS-ACE lipid nanoparticles containing rifampicin was analyzed for lyophilized particles kept in a tightly closed amber vial at refrigerator $\left(4 \pm 2^{\circ} \mathrm{C}\right)$ and room temperature $\left(25 \pm 2{ }^{\circ} \mathrm{C}\right)$ under a dark environment. The samples were taken at a predetermined time and the diameter size, polydispersity index, and rifampicin content were analyzed from the reconstituted suspension (Das et al., 2012; Kim et al., 2018).

\section{Cellular uptake of COS-ACE lipid nanoparticles}

Toxicity of the formulation was tested prior to cellular uptake analysis. It was determined against Vero and BALB/c 3T3 cells using the modified procedure previously described by Kumar et al. (2008). The level of $\mathrm{NADPH}^{+} \mathrm{H}^{+}$produced by viable cells was quantified using Alamar blue ${ }^{\circledR}$ by measuring the reduction of resazurin dye to resorufin (Borra et al., 2009). The cells were seeded into 96-well flat-bottom tissue culture plate at the amount of 1,000 cells/well and incubated in DMEM medium containing $10 \%$ FBS serum and $1 \%$ of antibiotic-antimicotic (Sigma Aldrich, Singapore) for 24 hours in a humidified atmosphere of $5 \% \mathrm{CO}_{2}$ at $37^{\circ} \mathrm{C}$. Nearly, $250 \mu \mathrm{l}$ of sample in complete medium - equal to $5 \mathrm{ppm}$ rifampicin — was added to each well. After 32 hours, the medium was changed with Alamar blue ${ }^{\circledR}$ in HBSS buffer and incubated for 1.5 hours at a temperature of $37^{\circ} \mathrm{C}$. The reduction dye by living cells was measured by spectrophotometer to calculate the difference absorption level at $600 \mathrm{~nm}$ for resazurin to $570 \mathrm{~nm}$ for resorufin. Cells without sample and cell incubated with phenol were analyzed as the positive and negative controls. 
Cellular internalization of COS-ACE lipid nanoparticles was analyzed using modified procedure from Gartziandia et al. (2015). BALB/c 3T3 cells was cultured in T75 flask, then the cells were split into confocal disc $\left(2 \times 10^{5}\right.$ cell/disc $)$ in $2 \mathrm{ml}$ DMEM medium containing 10\% FBS serum. Rifampicin loaded of NLC and COS-ACE lipid nanoparticles were labelled by nile red fluorochrome. In brief, the nile red solution $0.4 \% \mathrm{w} / \mathrm{w}$ was dissolved in ethyl acetate $(2 \mathrm{ml})$, then mixed into the oil phase. The water phase was mixed with the oil phase using homogenizer at $9,000 \mathrm{rpm}$ for 3 minutes. The mixture was sonicated for 3 minutes at the amplitude of $50 \mathrm{kHz}$. STPP solution was added dropwise to the homogenized nanoemulsion $(0.12 \%)$ and stirred using magnetic stirrer for 30 minutes. Before cellular uptake testing, the medium was discarded then the cells inside of the confocal disc were washed with PBS pH 7.42 times. The labelled NLC and COS-ACE lipid nanoparticles were diluted into $2 \mathrm{~mL}$ of serum-free medium to obtain a concentration of $50 \mu \mathrm{g} / \mathrm{ml}$, subsequently it was added to the disc and incubated for 30 minutes. The cells were then observed using confocal microscopy Fluoview FV10i at $60 \times$ magnification. Nile red dye was used with an excitation wavelength around $530 \mathrm{~nm}$ and the emission spectra wavelength of $607 \mathrm{~nm}$ was detected with a meta-detector scan range of 595-612 nm. Olympus image analysis software was employed for observing the cells.

\section{Statistical analysis}

Statistical analysis of the data was performed using a one-way analysis of variance (ANOVA) and differences between groups were tested by a one-way ANOVA with Tukey multiple comparison test using Minitab ${ }^{\circledR}$ 17. A P-value $<0.05$ was considered as significant. Correlation regression between two parameters were analyzed as a linear or quadratic model.

\section{RESULTS AND DISCUSSION}

\section{Synthesis of chitosan and acemannan conjugate}

Based on analysis, acemannan was successfully conjugated with chitosan through Schiff base reaction. This is shown by the wavelength shift of amide band of chitosan in the FTIR spectra. In the study, sodium triacetoxyborohydrate was used as a reducing agent to induce the reduction reaction of primary and secondary amine groups of chitosan. It was also used to induce ring opening of cyclic acemannan group to form free aldehyde groups. Subsequently, aldehyde groups reacted with the reduced amines of chitosan to form Schiff base ( $\mathrm{R}-\mathrm{CH}=\mathrm{N}-\mathrm{R}$ ) (AbdelMagid et al., 1996). Acetic acid was used as a proton donor in the reaction mixture of sodium triacetoxyborohydrate, chitosan and acemannan in tetrahydrofurane.

As shown in Figure 1, chitosan structure had primary amine at wavelength number of $1,637.56 \mathrm{~cm}^{-1}$. Following conjugation reaction with acemannan, the primary amine still existed at $1,629.85 \mathrm{~cm}^{-1}$ and an additional peak formed at $1,560.41$ $\mathrm{cm}^{-1}$ with the intensity ratio between the peaks of $1.07: 1 \%$. The new peak indicates wavelength shift from the primary amine to the secondary amine. The N-H bond at a wavelength of $3,412.08 \mathrm{~cm}^{-1}$ was also shifted to $3,431.26 \mathrm{~cm}^{-1}$ as a broader peak. Furthermore, a new peak appeared at $1,408.04 \mathrm{~cm}^{-1}$ indicating the existence of $\mathrm{C}=\mathrm{N}$ stretching modes. These results were similar with those found by Chaubey and Misra (2014), which showed changes of wavelength numbers when chitosan was conjugated with D-mannose. The molecular weight of COS and the COS-ACE conjugate, as determined by capillary viscometer, were $60.5 \pm 8.9$ $\mathrm{kDa}$ and $111.9 \pm 12.8$, respectively.

The free primary amine groups of the conjugate could still be found at the FTIR spectra. These positively-charged groups were expected to bind electrostatically with the negatively-charged of carboxylic acid and hydroxyl groups of lipid components in the NLC during the construction of COS-ACE lipid nanoparticles.

\section{Formulation of rifampicin-loaded COS-ACE lipid nanoparticles}

The success of antimicrobial therapies for intracellular infection mostly depends on the ability of the therapeutics to reach their designated intracellular target sites, while minimizing action and accumulation at non-specific sites (Rieux et al., 2006; van Vlerken et al., 2007). The nanoparticles were expected to mimic the entry process of pathogenic microbes with similarities in surface properties and particle size.In this study, the surface properties of the nanoparticles were designed to be similar to glycolipid structure of mycobacteria, with dimension of $0.2-0.5$ $\mu \mathrm{m}$ in width and 1-8 $\mu \mathrm{m}$ in length (Comas et al., 2010; Schlesinger et al., 2008). Some bacterial, fungal, and viral pathogens have surface glycolipid on their structure. Apart from surface property,

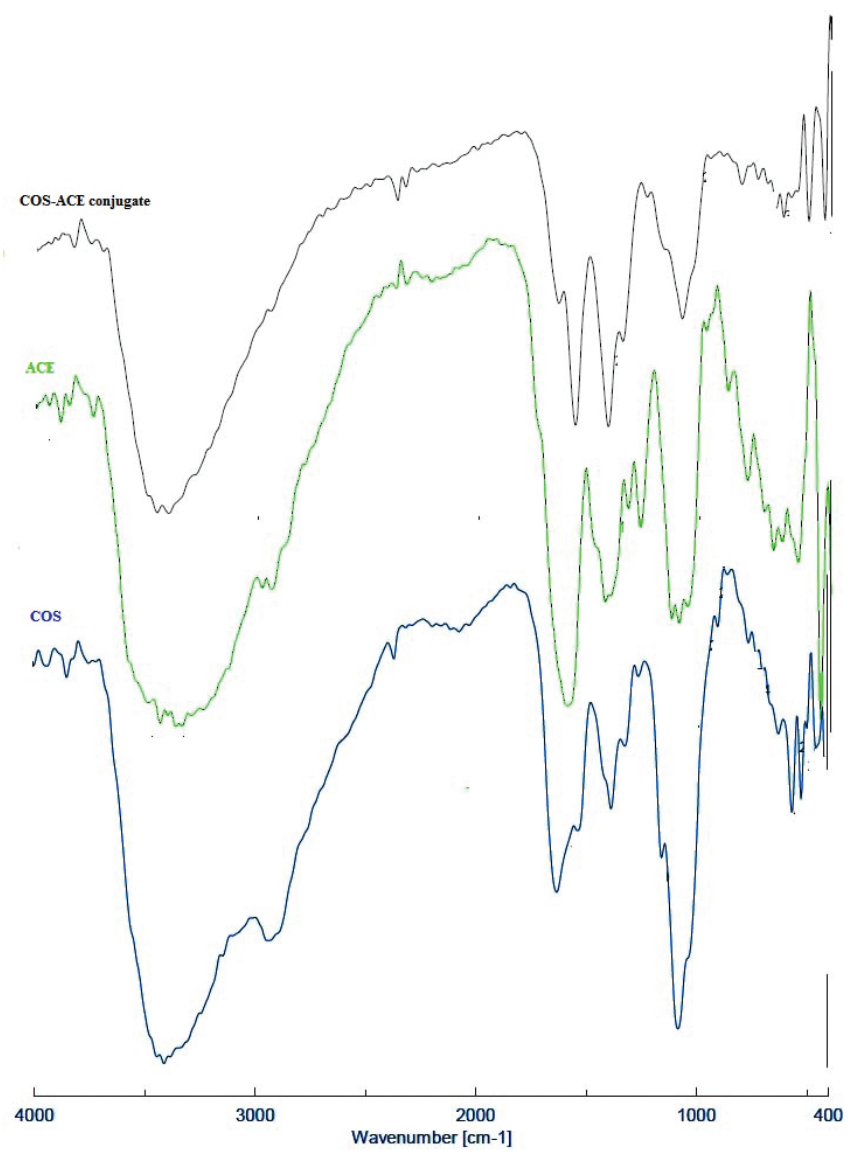

Figure 1. Infrared spectrum of (A) chitosan, (B) acemannan, and (C) COS-ACE conjugate. 
the particle size is another contributing factor for successful delivery. Considering the transfection mechanism into various cells such as macrophage, epithelial, and dendritic cells, particles smaller than $500 \mathrm{~nm}$ should be able to diffuse across biological membranes upon delivery (Rowe et al., 2006). Thus, the size of the nanoparticles was restricted to be in a range between 200 and $500 \mathrm{~nm}$. The size of NLC prior to COS-ACE conjugation was controlled not to exceed $200 \mathrm{~nm}$ to anticipate an increase in size upon the addition of COS-ACE conjugate in the final COS-ACE lipid nanoparticles formulation.

\section{Optimization of rifampicin-loaded NLC}

The emulsification-solvent evaporation method was chosen to prepare the NLC. The process was conducted at a room temperature to prevent degradation of rifampicin. Various factors were studied to observe the relation with particle size. The studied variables were as follows: the ratio of solid lipid and liquid lipid, the amount of Tween ${ }^{\circledR} 80$, the amount of rifampicin, the speed and time of homogenization, and the types and duration of sonication.

Cetyl alcohol $\left(\mathrm{MP}=45^{\circ} \mathrm{C}-56^{\circ} \mathrm{C}\right)$ and stearyl alcohol $\left(\mathrm{MP}=60^{\circ} \mathrm{C}-61^{\circ} \mathrm{C}\right.$ ) were used as solid lipid (Rowe et al., 2006; Sepulveda et al., 2003), while oleic acid as liquid lipid. Both lipids were mixed at various ratios to produce lipid admixture with melting point higher than $37^{\circ} \mathrm{C}$. Based on particle size analysis of the NLC, the optimum ratios of lipid to oleic acid to yield the intended size were 2:1 and 1:1 for cetyl alcohol and stearyl alcohol, respectively.

Homogenization was varied at the speed of $8,000 \mathrm{rpm}$ for 3,5 , and 10 minutes. Further, to obtain a smaller particle size, the NLC was processed using probe $(50 \mathrm{kHz})$ and water bath $(50 \mathrm{~Hz})$ sonicators for 2-6 minutes. It was shown that homogenization at $8,000 \mathrm{rpm}$ for 3 minutes, followed by sonication with probe sonicator for 3 minutes yield the most optimum size (117.8 \pm 4.2$)$ of the NLC (Fig. 2A). It was also shown that a longer duration of sonication did not reduce the particle size. Based on statistical analysis, there was no significant correlation between sonicator types and the duration of sonication $(p>0.05)$.

The size of NLC particles prepared using cetyl alcohol:oleic acid $(2: 1)$ was changed significantly at $3 \%-6 \% \mathrm{w} / \mathrm{v}$ of Tween ${ }^{\circledR} 80$ ( $\left.p=0.007\right)$. Meanwhile, surfactant concentrations showed less influence on the size of NLC particles made from stearyl alcohol:oleic acid (1:1) (Fig. 2B). All NLC prepared with stearyl alcohol-oleic acid $(1: 1)$ and $3-6 \% \% \mathrm{w} / \mathrm{v}$ Tween $\mathbb{R}$ 80 had particle size of less than $200 \mathrm{~nm}(p=0.414)$. While both stearyl alcohol and cetyl alcohol had the same required HLB (rHLB 15.5), cetyl alcohol had a lower molecular weight (MW of $242.44 \mathrm{~g} / \mathrm{mol}$ ) and melting point compared to stearyl alcohol (MW of $270.49 \mathrm{~g} / \mathrm{mol}, \mathrm{MP}: 60^{\circ} \mathrm{C}-61^{\circ} \mathrm{C}$ ). According to Oswald ripening phenomena, lower length of carbon chains in the lipid may dissolve partially in water resulting in particle aggregation (Taylor, 2003). The higher melting point of stearyl alcohol may lead to the higher cohesion force among the molecules resulting in a faster solidification of the NLC, thus preventing particle growth.

The addition of rifampicin at $0.5 \%-1.2 \% \mathrm{w} / \mathrm{v}$ to NLC prepared with stearyl alcohol:oleic acid $(1: 1)$ produced particles smaller than $200 \mathrm{~nm}$ in size. The size of the particle increased to $289.1 \pm 66.9 \mathrm{~nm}$ when added with $2 \% \mathrm{w} / \mathrm{v}$ rifampicin. Rifampicin concentration correlated linearly to particle size $(p=0.012)$ with the equation of $Y=90.52+98.41 X$ and $R^{2}$ of $62.4 \%$ (Fig. 2C). Larger particle size, which is 4-6 times higher, was observed from the NLC prepared with cetyl alcohol-oleic acid (2:1). When added with $0.5 \%$ rifampicin, the measured particle size of the NLC was
$\mathbf{A}$

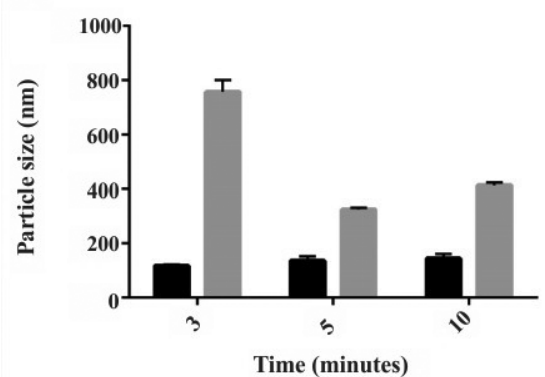

C

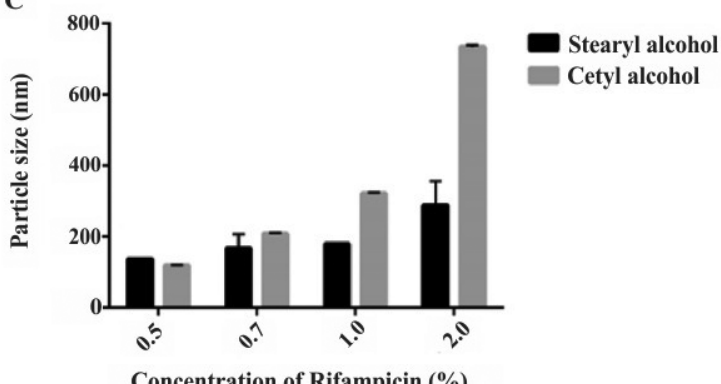

B

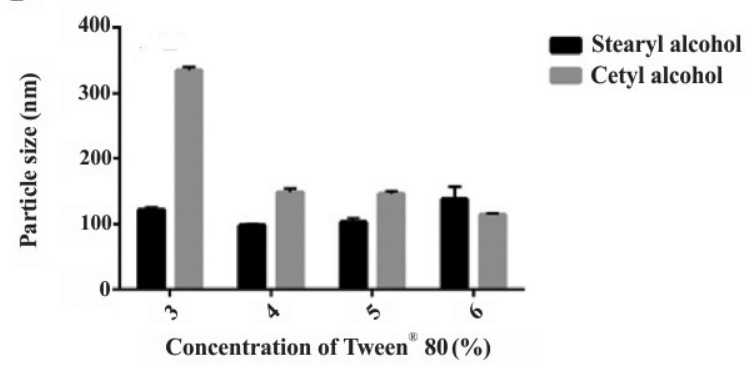

Regression model of various parameters:

\begin{tabular}{|c|c|c|c|}
\hline \multicolumn{4}{|c|}{ A. X: Duration of sonication (minute), Y: particle size (nm) } \\
\hline Sonicator type & Equation & $\mathrm{R}^{2}$ & $\mathbf{P}$ \\
\hline SP & $\mathrm{Y}=112.3+3.404 \mathrm{X}$ & $33.9 \%$ & 0.226 \\
\hline SW & $Y=715.7-36.15 X$ & 31.9 & 0.243 \\
\hline \multicolumn{4}{|c|}{ B. X:Tween ${ }^{\circledR} 80$ concentration (\%), Y:particle size (nm) } \\
\hline Lipid type & Equation & $\mathrm{R}^{2}$ & $\mathbf{P}$ \\
\hline Stearyl alcohol & $Y=89.96+5.69 X$ & $71.8 \%$ & 0.414 \\
\hline Cetyl alcohol & $\mathrm{Y}=484.9-66.34 \mathrm{X}$ & $72.5 \%$ & $\left.0.007^{\circ}\right)$ \\
\hline \multicolumn{4}{|c|}{ C. X:Rifampicin concentration (\%), Y:particle size (nm) } \\
\hline Lipid type & Equation & $\mathrm{R}^{2}$ & $\mathrm{P}$ \\
\hline Stearyl alcohol & $\mathrm{Y}=90.52+98.41 \mathrm{X}$ & $67.8 \%$ & $\left.0.012^{*}\right)$ \\
\hline Cetyl alcohol & $Y=82.05+408.1 X$ & $100 \%$ & $\left.0.000^{*}\right)$ \\
\hline
\end{tabular}

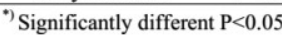

Figure 2. Particle size optimization of NLC at various parameters: (A) duration of sonication and sonicator type, (B) Tween 80 concentration and lipid type, and (C) rifampicin concentration and lipid type. 
$119.5 \pm 0.5 \mathrm{~nm}$ and increased to $734.6 \pm 4.4$ when added with $2 \%$ rifampicin. These parameters resulted in a strong linear correlation $(p=0.000)$, where $Y=82.05+408.1 X$, and $R^{2}$ of $100 \%$.

\section{Optimization of COS-ACE lipid nanoparticles preparation}

The COS-ACE lipid nanoparticles particles were prepared by using a one-step emulsification evaporation method. A cross-linker sodium tripoliphosphate, was added after the sonication process. Upon the addition of $1 \% \mathrm{w} / \mathrm{v}$ of COS-ACE conjugate to the previous NLC formulation, with homogenization at $8,000 \mathrm{rpm}$ for 3 minutes and sonication with probe sonicator for 3 minutes, the obtained particle size was $691.9 \mathrm{~nm}$. Thus, the speed and duration of homogenization were readjusted to compensate increasing viscosity and enlarging particles when polysaccharide conjugate was added.

The correlation between the speed and duration of homogenization with particle size followed quadratic models (Fig. 3A). However, there were no distinct pattern changes with variation of both factors. The longer duration of homogenization for 5 minutes at 8,000 and 10,000 rpm seems reduced the particle size, but an inconsistency data found at 9,000 rpm that produced the larger particles. This might be due to more collision among the particles for longer homogenization process may lead to particle aggregation. Therefore, we selected a moderate homogenization at 9,000 rpm for 3 minutes, which produced almost the smallest particle size.

The concentration of rifampicin was varied at $0.75 \%, 1 \%$, and $1.5 \%$ or equal to the theoretical drug loading of $33.3 \%, 40 \%$ and $50 \%$ for a total lipid of $0.75 \%$, and $20 \%, 25 \%$, and $33.3 \%$ for a total lipid of $1.5 \%$. Both total lipid concentrations followed linear model against rifampicin concentration (Fig. 3B). However, the increase in particle size at a total lipid of $1.5 \%$ was not significantly correlated with the increase of rifampicin concentration $(p=0.10)$. Surprisingly, COS-ACE lipid nanoparticles with total lipid of $0.75 \%$ had smaller particle size. With this formulation, rifampicin content equivalent to $40 \%$ theoretical drug loading resulted in $238 \pm 2 \mathrm{~nm}$ sized nanoparticles and the average size increased to $331.2 \pm 1.8 \mathrm{~nm}$ when rifampicin content was equivalent to $50 \%$ theoretical drug loading.

\section{Optimization of lyophilization process}

Since rifampicin is a relatively unstable compound in water, the NLC and nanoparticles were lyophilized to prevent rifampicin from hydrolysis. Lyophilization process by using liquid nitrogen $\left(-196^{\circ} \mathrm{C}\right)$ resulted in the smallest particle size for both the NLC and nanoparticles, with a slight increase from the freshly-prepared particles, but they were still in the desired size range (Fig. 4).

A very fast freezing process using liquid nitrogen produced small crystalline structures of ice crystals that dispersed the nanoparticles homogenously within the samples and therefore prevented particle aggregation in the next steps of the drying process. As a comparison, larger aggregates were resulted from moderate freezing in cold ethanol $\left(-40^{\circ} \mathrm{C}\right)$ and a significant particle growth $(p<0.05)$ was found in slow cooling in the freezer $\left(-20^{\circ} \mathrm{C}\right)$. A similar result was also found by Chung and colleagues (Chung et al., 2012), whom emphasized the use of liquid nitrogen for freeze drying process of nanoparticles. The lyophilized nanoparticles were suspended easily in water that might be due to stabilization effect provided by the positive charge of the COS-ACE conjugate.

\section{Physical properties and morphology analysis of COS-ACE lipid nanoparticles}

The obtained particle size of the COS-ACE lipid nanoparticles was in accordance with the targeted size, which
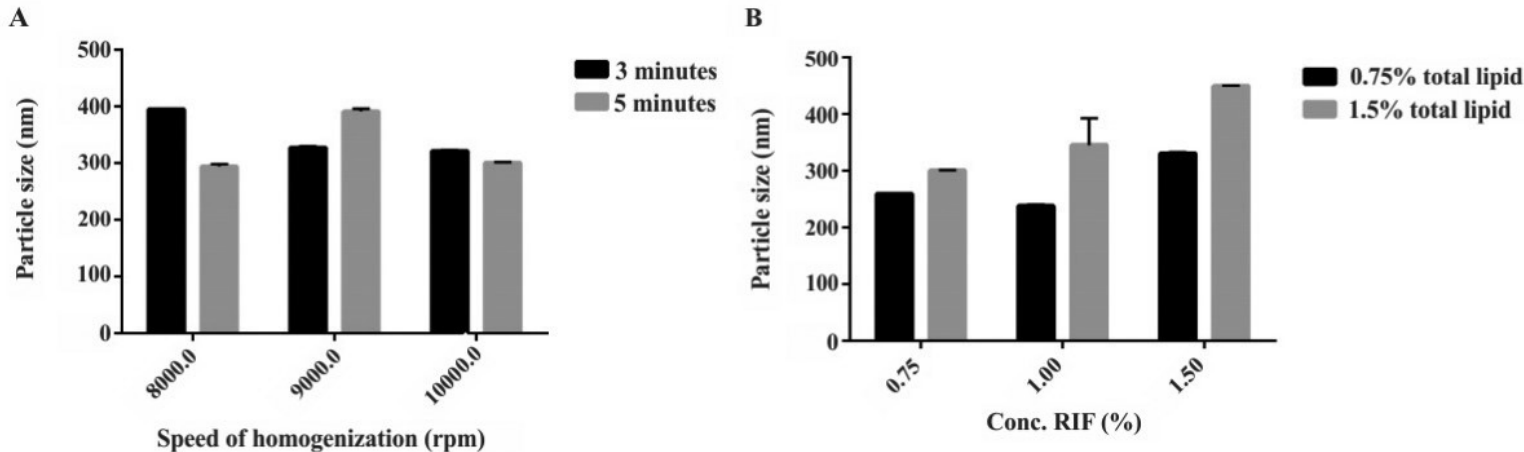

Regression model of various parameters:

\begin{tabular}{|c|c|c|c|}
\hline \multicolumn{4}{|c|}{ A. X: Speed of Homogenization (rpm), Y: particle size (nm) } \\
\hline Duration & Equation & $\mathrm{R}^{2}$ & P \\
\hline $3 \min$ & $Y=6112-1.29+0.000071 X^{2}$ & 99.3 & $0.000^{\circ}$ \\
\hline $5 \mathrm{~min}$ & $Y=2027-37 X+0.000020 X^{2}$ & 93.2 & $\left.0.012^{*}\right)$ \\
\hline \multicolumn{4}{|c|}{ B. X: Rifampicin concentration (\%), Y:particle size (nm) } \\
\hline Total Lipid & Equation & $\mathrm{R}^{2}(\%)$ & P \\
\hline $0.75 \%$ & $\mathrm{Y}=158.2+108.9 \mathrm{X}$ & 72.3 & 0.032 \\
\hline $1.5 \%$ & $Y=148.6+200.1 X$ & 84.0 & 0.10 \\
\hline
\end{tabular}

Figure 3. Particle size optimization of COS-ACE lipid nanoparticles at various parameters: (A) speed of homogenization and (B) concentration of the loaded rifampicin. 


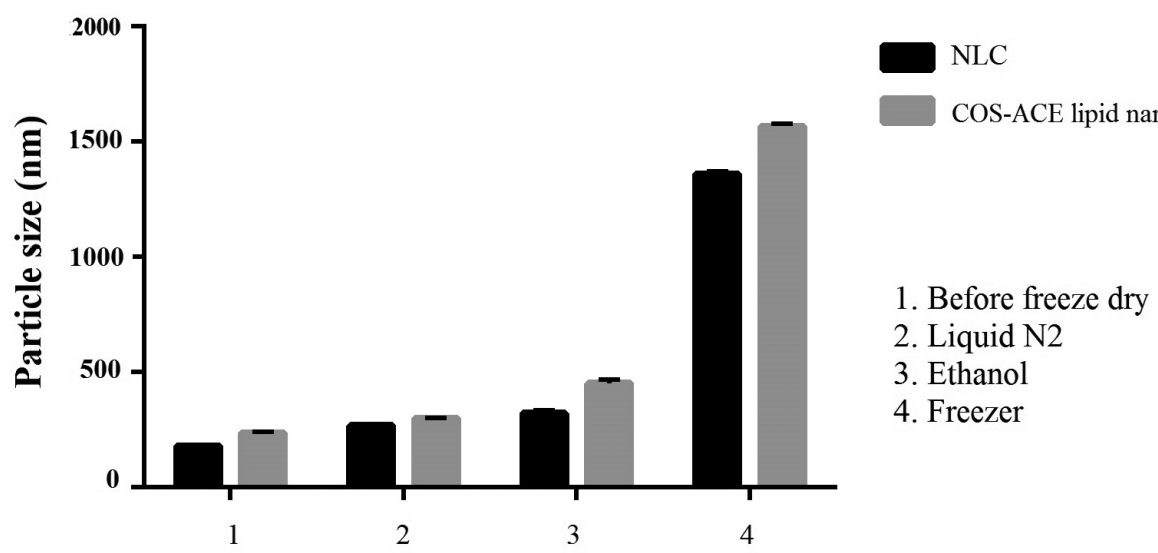

Figure 4. Particle size of freeze dried NLC and COS-ACE lipid nanoparticles, which were prepared by various speed of initial freezing prior to lyophilization process, namely: slow cooling in the freezer $\left(-20^{\circ} \mathrm{C}\right)$, moderate freezing in cold ethanol $\left(-40^{\circ} \mathrm{C}\right)$ and very fast freezing in liquid nitrogen $\left(-196^{\circ} \mathrm{C}\right)$. No-significant changes were found in liquid nitrogen pre-cooled treatment only $(p>0.05)$.

Table 1. Particle characteristics of the lyophilized NLC, chitosan nanoparticles, and COS-ACE nanocomposites.

\begin{tabular}{lccc}
\hline Parameters & NLC & Chitosan nanoparticles & COS-ACE nanocomposites \\
\hline Particle size $(\mathrm{nm})$ & $269.5 \pm 5.8$ & $206.8 \pm 10.91$ & $301.4 \pm 1.2$ \\
Polydispersity index & $0.317 \pm 0.42$ & $0.37 \pm 0.06$ & $0.28 \pm 0.02$ \\
Zeta potential $(\mathrm{mV})$ & $-4.48 \pm 2.089$ & $7.803 \pm 2.05$ & $1.66 \pm 1.39$ \\
EE $(\%)$ & $93.3 \pm 1.5 \%$ & $28.7 \pm 2.2 \%$ & $90.9 \pm 1.8 \%$ \\
\hline
\end{tabular}

All samples were analyzed in triplicate.

was between 0.2 and $0.5 \mu \mathrm{m}$ (Table 1 ). A low polydispersity index (PI) of the nanoparticles (0.28) showed that the globules were dispersed homogenously. The surface charge of the COSACE lipid nanoparticles was slightly positive/neutral $(+1.66 \pm$ 1.39). This might be due to the interaction between the negativelycharged of alcohol and carboxylic moieties of lipid components with the amine group of the protonated chitosan in COS-ACE conjugate. It can be predicted from data shown in Table 1 in which NLC and chitosan nanoparticles having zeta potential of $-4.48 \pm$ 2.089 and $+7.803 \pm 2.05$, which may interact during COS-ACE lipid nanoparticles formation.

The encapsulation efficiency of the nanoparticles was relatively good, which was above $90 \%$ (Table 1 ). It is found that the COS-ACE lipid nanoparticles formulation is a more effective delivery system for rifampicin, compared to chitosan nanoparticles that showed a low encapsulation efficiency. The high encapsulation efficiency may result from one-step incorporation of rifampicin within COS-ACE lipid nanoparticles. As the comparison for two-step preparation, the prior encapsulated drug at the first step may prone to lose during the second step of adsorption process. Previously, Jain et al. (2014) found less doxorubicin content in D-mannose modified solid lipid nanoparticles that were produced by adsorbing D-mannose on solidified NLC containing drug.

TEM imaging showed that the NLC had a spherical shape (Fig. 5A). Rifampicin was successfully entrapped within the NLC structure with no-obvious rifampicin particles observed outside the NLC. The preparation of the nanoparticles in a single step produced spherical COS-ACE lipid nanoparticles particles (Fig. 5C). It can be seen that the particles were distributed homogenously within the COS-ACE lipid nanoparticles. Furthermore, COS-ACE polymer chains were observed surrounding the lipid nanoparticles particles, which likely to stabilize the structure.

Confocal microscopy study also shows a similar characteristic of COS-ACE lipid nanoparticles in which COS-ACE chains occupied the outermost of NLC particles. Homogenously distributed red fluorescence intensity throughout the spherical NLC particles containing nile red fluorochrome (Fig. 5D and E) indicated that the particles were dominated by the lipids. As COS-ACE was incorporated into the nanoparticles, confocal imaging showed that the red fluorescence was concentrated in the middle of the particles and surrounded by a non-fluoresence layer (Fig. 5F and G). This can be explained by the steps involved to obtain COS-ACE lipid nanoparticles, where rifampicin and the lipid components were emulsified in COS-ACE-containing water. The lipid-soluble compounds spontaneously oriented their molecules to reside in the inner part of the particles, while COSACE conjugate - being relatively hydrophilic — prefer the aqueous environment. Since confocal microscopic analysis was conducted after the particles had been lyophilized, it can be assumed that this construction remained stable despite being exposed to the lyophilization process.

\section{Drug release and stability study}

In this research, the release of rifampicin from nanoparticles was analyzed at different $\mathrm{pH}$. The $\mathrm{pH} 7.4$ was selected to represent cytoplasmic environment, while $\mathrm{pH} 5$ represent phagosome condition when the naanoparticles enter the phagolysosome (Orfila, 1996). The amount of rifampicin released 

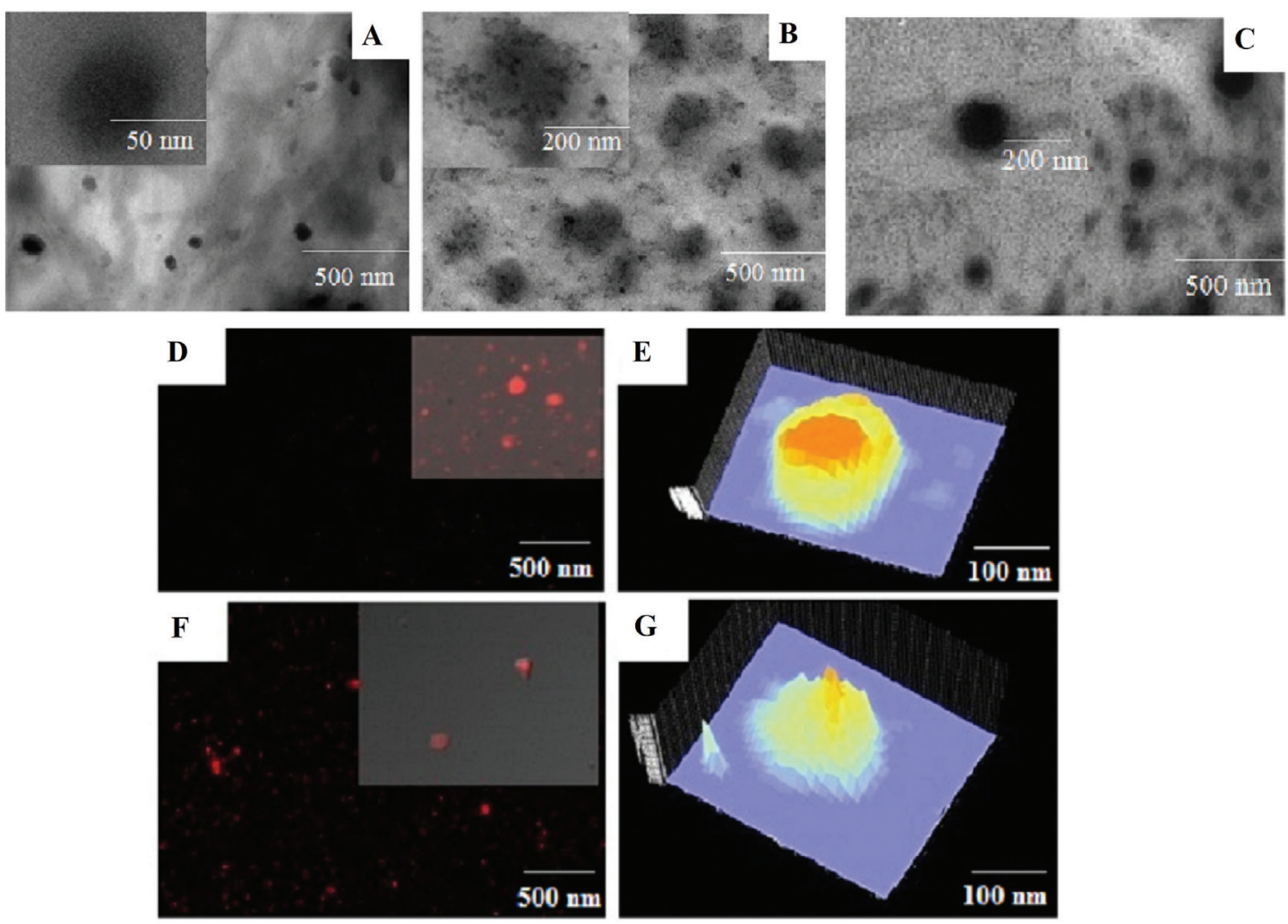

Figure 5. Morphology of nanoparticles revealed using transmission electron microscope and confocal microscope. TEM images of rifampicin-loaded in (A) NLC (B) chitosan nanoparticles (C) COS-ACE lipid nanoparticles. Confocal micrograph and fluorescence intensity of (D and E) nile red-rifampicin loaded in NLC and (F and G) COS-ACE lipid nanoparticles.

A



$\mathbf{C}$

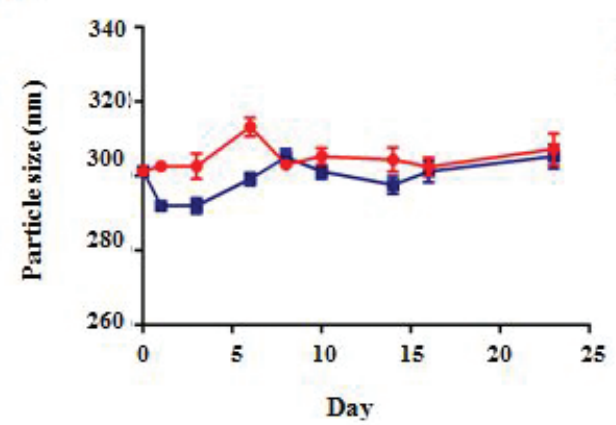

B

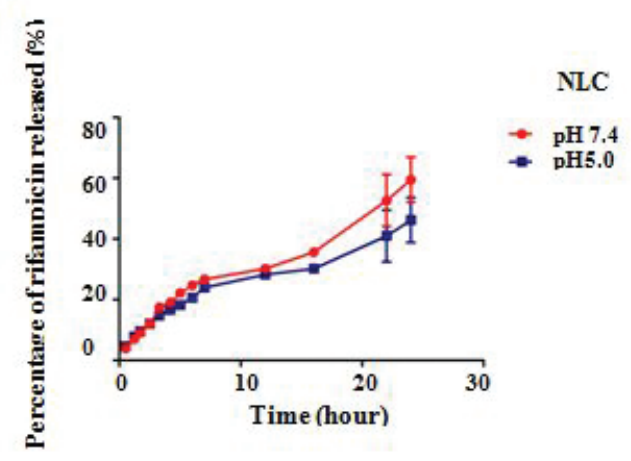

D

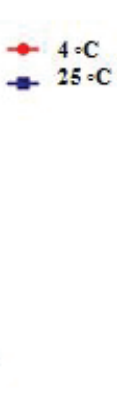

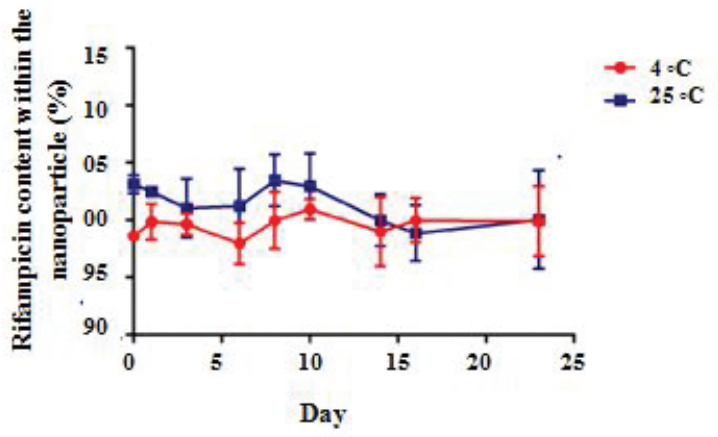

Figure 6. The percentage of rifampicin released at $\mathrm{pH} 5$ and 7.4 from the (A) COS-ACE lipid nanoparticles and (B) the NLC. Rifampicin released from COS-ACE lipid nanoparticles at $\mathrm{pH} 5$ was higher than at $\mathrm{pH} 7.4(p<0.05)$, while a similar release profile was found in NLC $(p>0.05)$. COS-ACE lipid nanoparticles were stable in terms of (C) particle size and (D) rifampicin content in the storage at refrigerator $\left(4 \pm 2^{\circ} \mathrm{C}\right)$ and room temperature $\left(25 \pm 2^{\circ} \mathrm{C}\right)$. 
from the nanoparticles at $\mathrm{pH} 5$ was higher than the amount released at $\mathrm{pH} 7.4$ (Fig. 6A). At pH 5, chitosan is protonated thus becomes highly soluble in PBS. This result indicates minimum extracellular premature release of rifampicin at $\mathrm{pH} 7.4$ and higher intracellular release. A different release profile was shown by NLC where a similar release rate of rifampicin at $\mathrm{pH} 7.4$ and at $\mathrm{pH} 5(p>0.05$, Fig. 6B).

The COS-ACE lipid nanoparticles were stable up to the last day of the stability testing study, in terms of the particle size and content after reconstitution, both at refrigerator temperature $\left(3^{\circ} \mathrm{C}-4^{\circ} \mathrm{C}\right)$ and room temperature $\left(25^{\circ} \mathrm{C}-26^{\circ} \mathrm{C}\right)$. The particle size of the nanoparticles during storage at both temperatures was in the average of $302.16 \pm 5.12 \mathrm{~nm}$ with a polydispersity index of $0.25 \pm$ 0.04 (Fig. 6C). The rifampicin content in refrigerator and room temperature storage was also no-significant change at $90 \%-100 \%$ (Fig. 6D). The results between two storage conditions did not show a significant difference.

\section{In-vitro cellular uptake}

Intracellular trafficking of a number drugs using specific delivery system has been shown in various cell lines, such as epithelial cells (Vaysse et al., 2004; Zhang et al., 2014) and fibroblast cells (Vaysse et al., 2004; Zhu et al., 2009). Furthermore, a wide range of cell type was subjected for pathogen intracellular invasion (Drobniewski et al., 2009; Ellington et al., 2006; Forret and Tamura, 2010). Thus, a variety of cells are proposed to become candidate targets of the COSACE lipid nanoparticles developed in this research. One of the target cells tested for cellular uptake here was fibroblast cell, which is a potential target of intracellular $S$. aureus infection through skin invasion. Clinically, it can be found in diabetic foot ulcer in which extracellular $S$. aureus initially infects fibroblast in wound area. Due to impairment of host defense mechanism caused by low blood flow to the lower extremities, the bacteria cannot be eradicated and likely to grow inside host cells as latent infection in chronic wound or even invading further to bone causes osteomyelitis (Honeyman et al., 2001). Limited blood perfusion consequently will limit the supply of professional and non-professional phagocytic cells having CLRs as the primary target cells of this delivery. Therefore, we tested the internalization of acemannan modified lipid nanoparticles in fibroblast cells (BALB/c 3T3 cells).

The COS-ACE lipid nanoparticles was shown to be non-toxic when tested against Vero and BALB/c 3 T3 cells where the percentage of viable cells were $92.8 \pm 4.25$ and $95.0 \pm 1.13$, respectively. No significant change was found when compared to viability of positive control without treatment, which were $100 \pm$ 3.03 and $99.4 \pm 4.12(p>0.05)$. The negative control of phenolincubated cells could not be observed since almost all the cells had undergone lysis. A very weak positive charge of COS-ACE lipid nanoparticles $(1.66 \mathrm{mV})$ did not provide negative effect to the cell integrity. This may induce cell adhesion required in the cell penetration process for intracellular transport to cytoplasm (Sheng et al., 2016).

The nile red fluorochrome labelled of COS-ACE lipid nanoparticles containing rifampicin (NC-NR-RIF) were internalized by BALB/c 3 T3 cells after 30 minutes of incubation, as shown in Fig. 7A. Nanoparticles were observed around the cytoplasmic area of the cells. Outside of the cells, the emission intensity of NC-NR-RIF was low and became higher when entering the cytoplasmic region. The emission intensity was decreased again when entering the nucleus and increased further around the cytoplasmic area. It seems the particles did not enter the nucleus. A similar result was also observed in NLC-NR-RIF samples (Fig. 7B) but with a lower intensity compared to COSACE lipid nanoparticles. These results proved that the formation of glycolipid structure as surface modified NLC using acemannan at the size of $0.2-0.5 \mu \mathrm{m}$ could improve cellular transport of nanoparticle.

Previous researches have shown the success of cell internalization of lipid carriers (Bhise et al., 2018; Chuan et al., 2013; Pandey and Ahmad, 2011), lipid nanoparticles coated with COS (Gartziandia et al., 2015; Zhang et al., 2014) or D-mannose-surface-modified (Song et al., 2015). D-mannosemodified dendrimer or chitosan nanoparticles for intracellular targeting have also been addressed by previous research (Chaubey and Misrha, 2014; Kumar et al., 2008). However, the use of acemannan modified nanocarrier for intracellular targeting has not been studied yet. Acemannan has been shown to induce the expression of some surface receptors on murine macrophage RAW 264.7, which involved in the phagocytosis process, such as CD11a, CD18, and mannose receptor, and a specific receptor mediated pathway (Simõesa et al., 2012; Zhang et al., 1996). Acemannan has also been reported to induce nitric oxide and cytokine production, such as IL-6 and TNF-a (Pandey and Ahmad, 2011). Furthermore, this acetylated polymannose has also been known for promoting differentiation of immature dendritic cells (Lee et al., 2001). These activities are important to facilitate internalization of antibiotic in COS-ACE-NLC lipid nanoparticles delivery system for optimizing eradication of the intracellular pathogen. In the future research, the extent of internalization of antibiotic encapsulated within nanoparticles against macrophages and dendritic cells will be quantified at various ratios of lipid to COS-ACE conjugate and composite concentrations.

\section{CONCLUSION}

The present work establishes suitability of NLC modified with chitosan-acemannan conjugate as a drug delivery system for intracellular targeting of rifampicin. The COS-ACE lipid nanoparticles were successfully prepared by a one-step preparation process. The conjugate deposited on NLC surface by electrical binding and provided steric stabilization of the lipid globules during nanoparticle solidification and drying. The resulted COSACE lipid nanoparticles showed homogenous size at around 300 $\mathrm{nm}$, with narrow polydispersity index and positive zeta potential $(1.66 \pm 1.39 \mathrm{mV})$. A good solubility of rifampicin in the selected lipid blend - a mixture of oleic acid and stearyl alcohol 1:1resulted in a high entrapment efficiency of more than $90 \%$. Cell viability studies using Vero cells and BALB/c 3T3 cells revealed no toxicity of the nanoparticles. Furthermore, cellular uptake of rifampicin-loaded COS-ACE lipid nanoparticles was shown in BALB/c 3T3 cells. To conclude, the developed formulation is a promising carrier for intracellular transport of lipid soluble antibiotics. 

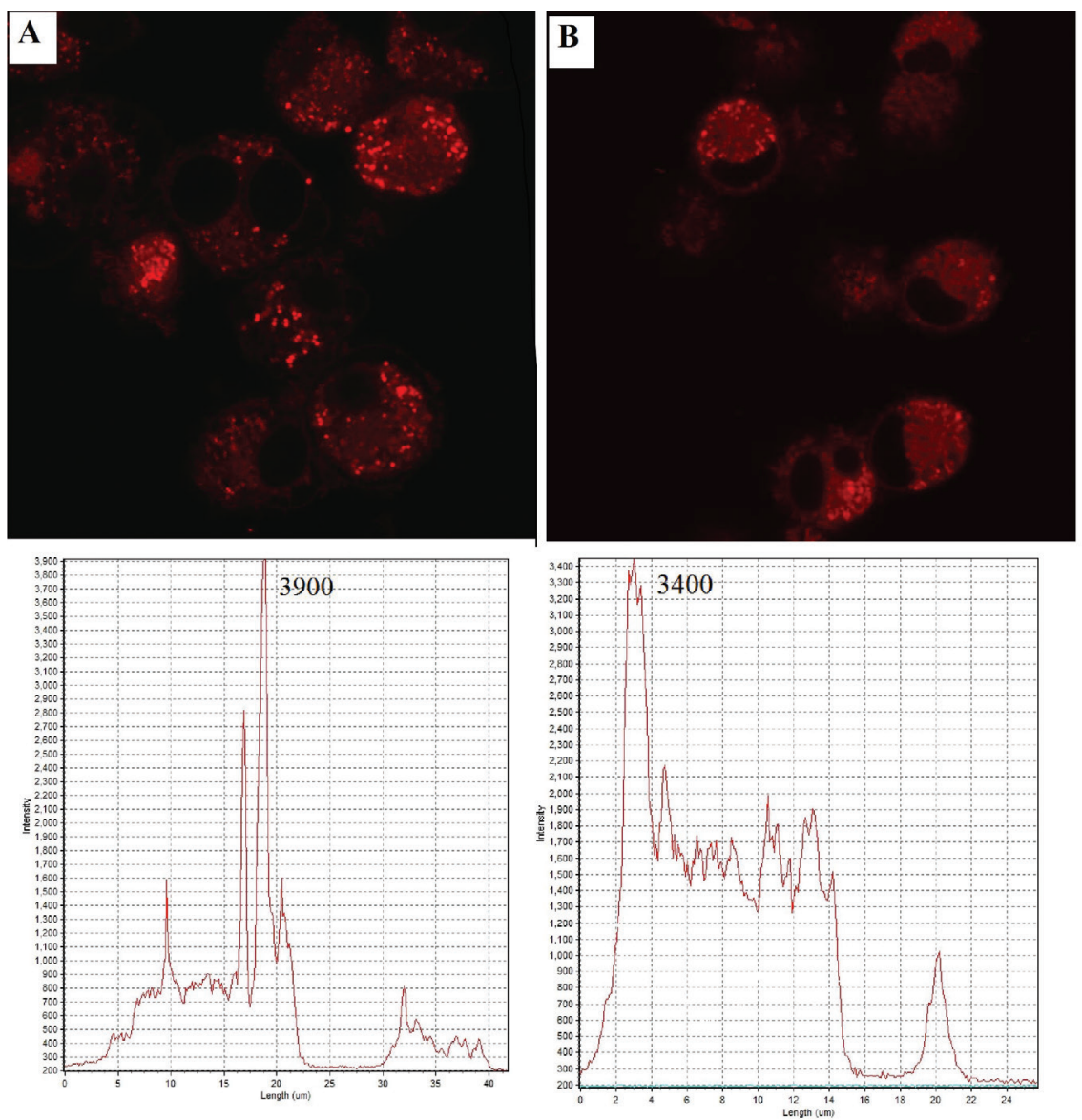

Figure 7. Confocal images of intracellular targeting profiles of nile red and rifampicin loaded nanoparticles of (A) COS-ACE lipid nanoparticles (NC-NR-RIF) and (B) NLC particles (NLC-NR-RIF) on BALB/c 3T3 cells after 30 minutes of incubation. The cell uptake of COS-ACE lipid nanoparticles particles was higher compared with NLC shown by higher intensity of fluorescence.

\section{ACKNOWLEDGMENTS}

This research was funded by "Desentralisasi" research grant, The Ministry of Research Technology and Higher Education of the Republic of Indonesia. Chito-olygosaccharide (COS) used in this work was produced using research grant of Development of Raw Materials for API/excipient and Traditional Medicines, Directorate General of Pharmaceutical and Medical Devices, The Ministry of Health of the Republic of Indonesia. We are grateful to Ms. Fatma Ayatiliulil Albab and Dewi Satwika from PT. Fajar Mas Murni for their assistance in confocal microscope imaging.

\section{CONFLICT OF INTEREST}

The authors declare no conflict of interest.

\section{REFERENCES}

Abdel-Magid AF, Carson KG, Harris BD, Maryanoff CA, Shah RD. Reductive amination of aldehydes and ketones with sodium triacetoxyborohydride. Studies on direct and indirect reductive amination procedures. J Org Chem, 1996; 61(11):3849-62.

Armstead AL, Li B. Nanomedicine as an emerging approach against intracellular pathogens. Int J Nanomedicine, 2011; 6(1):3281-93.

Bhise K, Sau S, Kebriaei R, Rice S, Stamper K, Alsaab H, Rybak MJ, Iyer A. Combination of vancomycin and cefazolin lipid nanoparticles for overcoming antibiotic resistance of MRSA. Materials, 2018; 11(7):1245.

Borra RC, Lotufo MA, Gagioti SM, Barros FDM, Andrade PM.
A simple method to measure cell viability in proliferation and cytotoxicity assays. Braz Oral Res, 2009; 23(3):255-62.

Chaubey P, Mishra B. Mannose-conjugated chitosan nanoparticles loaded with rifampicin for the treatment of visceral leishmaniasis. Carbohydr Polym, 2014; 101:1101-8.

Chuan J, Li Y, Yang L, Sun X, Zhang Q, Gong T, Zhang Z. Enhanced rifampicin delivery to alveolar macrophages by solid lipid nanoparticles. J Nanopart Res, 2013; 15:1634-43.

Chung N-O, Lee MK, Lee J. Mechanism of freeze-drying drug nanosuspensions. Inter J Pharm, 2012; 437(1-2):42-50.

Comas I, Chakravartti J, Small PM, Galagan J, Niemann S, Kremer K, Ernst JD, Gagneux S. Human T cell epitopes of Mycobacterium tuberculosis are evolutionarily hyperconserved. Nature Genet, 2010; 42(6):498-503.

Das S, Ng WK, Tan RB. Are nanostructured lipid carriers (NLCs) better than solid lipid nanoparticles (SLNs): development, characterizations and comparative evaluations of clotrimazole-loaded SLNs and NLCs?. Eur J Pharm Sci, 2012; 47(1):139-151.

Drobniewski FA, Amin AK, Balabanova Y. Non-pulmonary tuberculosis and mycobacterial infection. Medicine, 2009; 37(12):649-53.

Ellington JK, Harris M, Hudson MC, Vishin S, Webb LX, Sherertz R. Intracellular Staphylococcus aureus and antibiotic resistance: implications for treatment of staphylococcal osteomyelitis. J Orthop Res, 2006; 24(1):87-93.

Faville RJ, Zaske DE, Kaplan EL, Crossley K, Sabath LD, Quie PG. Staphylococcus aureus endocarditis: combined therapy with vancomycin and rifampicin. JAMA, 1978; 240(18):1963-5. 
Forrest GN, Tamura K. Rifampin combination therapy for nonmycobacterial infections. Clin Microbiol Rev, 2010; 23(1):14-34.

Gartziandia O, Herran E, Pedraz JL, Carro E, Igartua M, Hernandeza RM. Chitosan coated nanostructured lipid carriers for brain delivery of proteins by intranasal administration. Colloid Surf B, 2015; 134:304-13.

Goy RC, Britto Dd, Assis OBG. A review of the antimicrobial activity of chitosan. Polímeros: Ciência e Tecnologia, 2009; 19(33):241-7.

Honeyman A, Friedman $\mathrm{H}$, and Bendinelli $M$ (eds.). Staphylococcus aureus infection and disease. Springer Science \& Business Media, New York, NY, pp 213-67, 2001.

Jain V, Gupta A, Pawar VK, Asthana S, Jaiswal AK, Dube A, Chourasia MK. Chitosan-assisted immunotherapy for intervention of experimental leishmaniasis via amphotericin B-loaded solid lipid nanoparticles. Appl Biochem Biotechnol, 2014; 174(4):1309-30.

Jørgensen NP, Hansen K, Andreasen CM, Pedersen M, Fuursted $\mathrm{K}$, Meyer RL, Petersen E. Hyperbaric oxygen therapy is ineffective as an adjuvant to daptomycin with rifampicin treatment in a murine model of Staphylococcus aureus in implant-associated osteomyelitis. Microorganisms, 2017; 5(2):pii: E21.

Kasaai MR, Arul J, Charlet G. Intrinsic viscosity-molecular weight relationship for chitosan. J Polym Sci Part B Polym Phys, 2000; 38(19):2591-8.

Kerrigan AM, Brown GD. C-type lectins and phagocytosis. Immunobiology, 2008; 214:562-75; doi: 10.1016/j.imbio.2008.11.003

Kim HI, Park S, Park S, Lee J, Cho K, Jee JP, Jang DJ. Development and evaluation of a reconstitutable dry suspension to improve the dissolution and oral absorption of poorly water-soluble celecoxib. Pharmaceutics, 2018; 10(3):140.

Kumar PV, Asthana A, Dutta T, Jain NK. Intracellular macrophage uptake of rifampicin loaded mannosylated dendrimers. J Drug Target, 2008; 14(8):546-56.

Lee JK, Lee MK, Yun Y-P, Kim Y, Kim JS, Kim YS, Kim K, Han $\mathrm{SS}$, Lee C-K. Acemannan purified from Aloe vera induces phenotypic and functional maturation of immature dendritic cells. Int Immunopharm, 2001; 1(7):1275-84.

Naglik JR, Moyes D. Epithelial cell innate response to Candida albicans. Adv Dent Res, 2011; 23(1):50-5.

Neyrolles O, Guilhot C. Recent advances in deciphering the contribution of Mycobacterium tuberculosis lipids to pathogenesis. Tuberculosis, 2011; 91(3):187-95.

Orfila J. Definition of intracellular pathogens. Clin Microbiol Infect, 1996; 1:S1-S2.

Patel BK, Parikh RH, Aboti PS. Development of oral sustained release rifampicin loaded chitosan nanoparticles by design of experiment. J Drug Deliv, 2013; 2013:1-10.

Pandey R, Ahmad Z. Nanomedicine and experimental tuberculosis: facts, flaws, and future. Nanomed Nanotech Biol Med, 2011; 7(3):259-72.

Perlroth J, Kuo M, Tan J, Bayer AS, Miller LG. Adjunctive use of rifampin for the treatment of Staphylococcus aureus infections. Arch Intern Med, 2008; 168(8):805-19.

Rowe RC, Sheskey PJ, Weller PJ (eds.). Handbook of pharmaceutical excipients Pharmaceutical press, London, UK, , Volume 6, pp 150-1, 2006.
Rieux AD, Fievez V, Garinot M, Schneider Y-J, Préat V. Nanoparticles as potential oral delivery systems of proteins and vaccines: a mechanistic approach. J Control Release, 2006; 116(1):1-27.

Schlesinger LS, Azad AK, Torrelles JB, Esteban Roberts, Vergne I, Deretic V. Handbook of tuberculosis: immunology and cell biology. WILEY-VCH Verlag GmbH \& Co. KGaA, Weinheim, 2008.

Sepulveda E, Kildsig DO, Ghaly ES. Relationship between internal phase volume and emulsion stability: the cetyl alcohol/stearyl alcohol system. Pharm Dev Technol, 2003; 8(3):263-75.

Sheng R, Zhuang X, Wang Z, Cao A, Lin K, Zhu JX. Cationic nanoparticles assembled from natural-based steroid lipid for improved intracellular transport of siRNA and pDNA. Nanomaterials, 2016; 6(4):69-87.

Simõesa J, Nunesb FM, Dominguesa P, Coimbraa MA, Dominguesa MR. Mass spectrometry characterization of an Aloe vera mannan presenting immunostimulatory activity. Carbohydr Polym, 2012; 90(1):229-36.

Song X, Lin Q, Guo L, Fu Y, Han J, Ke H, Sun X, Gong T, Zhang Z. Rifampicin loaded mannosylated cationic nanostructured lipid carriers for alveolar macrophage-specific delivery. Pharm Res, 2015; 32(5):1741-51.

Taylor P. Ostwald ripening in emulsions: estimation of solution thermodynamics of the disperse phase. Adv Colloid Interface Sci, 2003; $106(1-3): 261-85$

van Vlerken LE, Vyas TK, Amiji MM. Poly(ethylene glycol)modified nanocarriers for tumor-targeted and intracellular delivery. Pharm Res, 2007; 24(8):1405-14.

Vaysse L, Harbottle R, Bigger B, Bergau A, Tolmachov O, Coutelle C. Development of a self-assembling nuclear targeting vector system based on the tetracycline repressor protein. J Biol Chem, 2004; 279(7):5555-64

Xie S, Tao Y, Pan Y, Qu W, Cheng G, Huang L, Chen D, Wang X, Liu Z, Yuan Z. Biodegradable nanoparticles for intracellular delivery of antimicrobial agents. J Control Release, 2014; 187:101-17.

Zhang L, Tizard IR. Activation of a mouse macrophage cell line by acemannan: the major carbohydrate fraction from Aloe vera gel. Immunopharmacology, 1996; 35(2):119-28.

Zhang W, Liu J, Zhang Q, Li X, Yu S, Yang X, Kong J, Pan W. Enhanced cellular uptake and anti-proliferating effect of chitosan hydrochlorides modified genistein loaded NLC on human lens epithelial cells. Int J Pharm, 2014; 471(1-2):118-26.

Zhu WL, Shin SY. Antimicrobial and cytolytic activities and plausible mode of bactericidal action of the cell penetrating peptide penetratin and its lys-linked two-stranded peptide. Chem Biol Drug Des, 2009; 73(2):209-15.

How to cite this article:

Suciati T, Rachmawati P, Soraya E, Mahardhika AB, Satrialdi, Hartarti R, Anggadiredja K. A novel acemannan-chitosan modified lipid nanoparticles as intracellular delivery vehicles of antibiotic. J App Pharm Sci, 2018; 8(12): 001-011. 